\title{
Frontiera e convivenza nell'opera di Fulvio Tomizza
}

Riassunto: In questo intervento si cercherà di affrontare il tema dell'identità di frontiera in Fulvio Tomizza, prendendo in considerazione in particolare alcuni romanzi e scritti dell'autore. Nell'analisi si cercherà di analizzare in particolar modo il rapporto dello scrittore con la sua terra natale quale simbolo di identità. Si tratta di un'identità complessa, in quanto il territorio in cui Tomizza vive, istriano prima e triestino poi, è composto da più culture ed etnie, che da sempre si intrecciano e convivono: un territorio metafora dell'identità dello scrittore stesso. Ed è proprio nell'unione, nell'intreccio, che lo scrittore vede l'unica soluzione possibile per giungere ad una costruttiva convivenza in questa regione, quasi un'allegoria del mondo a venire. Il grande merito dello scrittore, infatti, è stato quello di aver saputo comprendere le ragioni dell' 'altro' in questo territorio, in cui spesso prevalevano conflitti e recriminazioni.

\section{Borders and cohabitation in the work of Fulvio Tomizza}

Abstract: This paper will analyse the theme of border identity in Fulvio Tomizza, taking into account some of the author's novels and writings and focusing in particular on his relationship with his native land as a symbol of identity. That is in fact a complex identity, since the territory in which Tomizza lived, first Istria and then Trieste, is made up of several cultures and ethnic groups, which have always intertwined and coexisted: a territory that is a metaphor for the writer's identity. It is precisely in the blend, in the interweaving, that the writer sees the only possible solution to achieve a constructive coexistence in this region, almost an allegory of the world to come. The great merit of the writer, in fact, was to understand the point of view of the 'other' in this territory, where conflicts and recriminations often prevailed. 


\section{Introduzione}

Quando si affronta il discorso dell'Istria emerge sempre il problema dell'appartenenza etnica, tema che Tomizza affronta in più opere e di cui si occupò durante tutto il suo percorso di scrittore. L'Istria delle opere di Tomizza è una terra di contadini, immersa in una condizione dialettale, etnicamente e culturalmente mista, una terra che ha visto passare diverse dominazioni, tra le quali, in tempi recenti, austriaci, italiani, tedeschi e infine il comunismo di Tito.

In tutte le opere dello scrittore emerge lo spirito della terra istriana, con le sue luci e le sue ombre, la vicenda travagliata di una terra di confine, una terra di incontro e scontro nella storia di stati, popoli, culture, lingue e tradizioni diverse. In questo ambiente si colloca il concetto di identità di frontiera che, in molti casi, può rivelarsi di estrema complessità come riferirà lo stesso scrittore nei suoi romanzi. Fulvio Tomizza infatti crea personaggi con caratteristiche di individui aventi un'identità legata a più connotazioni culturali ed etniche. Una condizione che impedisce al singolo di esprimere la propria identità in modo definito e distinto, anche se a volte egli viene posto in una condizione di scelta che potrebbe portarlo alla negazione dei suoi tratti essenziali, etnici e/o culturali. Tuttavia è proprio questo individuo che si rivela pronto al confronto e all'assimilazione delle diversità per poter infine comprendere ed accettare consapevolmente la propria natura multiculturale.

Per la stesura di questo articolo sono stati presi in esame alcuni romanzi di Tomizza che descrivono luoghi in cui lo scrittore ha vissuto, ma soprattutto danno lo spunto per comprendere l'idea di identità plurima, a lui tanto cara, come unica idea di sopravvivenza pacifica per queste terre di confine. Inoltre sono state analizzate altre sue opere significative, nelle quali è l'autore stesso a spiegare le ragioni delle sue scelte, come Alle spalle di Trieste e Destino di frontiera.

L'indagine si propone pertanto di puntualizzare ed esporre il modo in cui il concetto di 'identità di frontiera' viene espresso in questi testi e quali effetti esso produce nell'ambito della convivenza tra culture ed etnie compresenti 


\section{La terra natale come simbolo di identità}

In una terra come l'Istria che nei secoli è stata governata da diversi 'padroni', prima i Romani poi la Repubblica veneta, poi l'Impero austro-ungarico, poi l'Italia, l'Italia fascista, e poi la Jugoslavia e il regime di Tito, e che vede intersecarsi sul suo territorio popolazioni diverse, miste, non vi è soltanto il problema della definizione dei confini, ma anche, molto forte, quello dell'appartenenza etnica. In questa terra, ma anche in tutte le altre zone di confine, in cui negli individui sono presenti più etnie e si parlano più lingue, molto spesso gli abitanti non si possono identificare con una sola patria ben definita. Di solito in queste persone, per non essere costrette a dover scegliere tra due culture o due etnie, affiora l'idea di appartenere a un mondo più vasto che comprenda più culture e più etnie, o addirittura, per non far torto a nessuno e rimanere imparziali, si arriva ad un sentimento di inappartenenza. ${ }^{2}$

Questo è quanto è successo anche a Tomizza, che sceglierà di appartenere ad entrambe le culture, quella italiana e quella slava, ${ }^{3}$ nella speranza, un po' utopica e un po' sentimentale (come dirà lui stesso), che un giorno esse possano convivere non soltanto in lui, ma in tutta la popolazione istriana. ${ }^{4}$ Lo scrittore fa questa scelta, che è tra l'altro una scelta del tutto legittima, visto che suo padre, Ferdinando Tomizza, era di sentimenti italiani, mentre la madre, Margherita Frank Trento, era invece di origine slovena. Inoltre, egli trascorse la sua infanzia nel paesino natale di Materada, un borgo che si trova tra Umago e Buie, in cui da sempre convivevano in armonia popolazioni di diversa origine etnica. Da questa sua coscienza multietnica, che diventerà una questione di cultura, ma anche e soprattutto di tradizione storica alla ricerca delle proprie origini, in cui identificarsi e riconoscersi per determinare un punto di riferimento per la propria esistenza, prendono il via i temi portanti di tutte le opere di Tomizza, quelli della frontiera, a partire dal primo romanzo Materada (1960), fino all'ultimo, postumo, Sogno dalmata (2001). Si tratta però di una frontiera che non coincide con $\mathrm{i}$ confini di stato, ma, come si vede in Materada, sarà più un conflitto tra mondi

1 Fulvio Tomizza, Alle spalle di Trieste, Milano, Bompiani, 2009, p. 124. La gente dell'Istria ha sempre considerato come 'padrone' colui che è al potere.

2 Angelo Ara e Claudio Magris, Trieste. Un'identità di frontiera, Torino, Einaudi, 2007, p. 192.

3 Il termine «slava» è stato qui usato per definire le due culture slave che lo scrittore considera come sue, quella slovena e quella croata, e non ha quella caratteristica peggiorativa che di solito gli italiani della zona limitrofa attribuiscono agli sloveni (o ai croati) per definirli di cultura inferiore.

4 F. Tomizza, Alle spalle di Trieste, cit., p. 194. 
etnici differenti, che farà nascere ostilità generate da ideologie diverse, che porteranno poi all'esodo di coloro che non si metteranno dalla parte 'giusta'.

La successione storica dei regimi, prima fascista e poi comunista, alimentarono la frattura dell'Istria a più livelli: culturale, etnico e geografico. La lingua divenne punto focale di rivalità tra gruppi etnici, e separò in due fazioni contrapposte la popolazione, inducendo la gente a scegliere un'etnia, una nazione, un linguaggio, sviluppando così il processo di annientamento del modello multietnico omogeneo che nei secoli era stato proprio dell'Istria. Questo mondo così unito, come lo ricorda lo scrittore, ${ }^{5}$ aveva subito una prima pressione verso la fine dell'Ottocento, quando improvvisamente nacque il sentimento nazionale, portato dai parroci e dai maestri che venivano nel suo paese da fuori. Questi, a seconda dell'appartenenza all'etnia croata o a quella italiana, cercavano di influenzare le persone a schierarsi da una parte o dall'altra. Secondo Tomizza non si tratta di «conformismo banale», ma di «legittima difesa» da parte di un popolo che sente che, per ragioni di sopravvivenza, ci si debba schierare con chi detiene il potere. ${ }^{6} \mathrm{E}$ sicuramente una risposta rassegnata che esprime sfiducia nelle istituzioni, nei regimi, nelle ideologie e anche in quelli che agitano tali ideologie.

In Tomizza, invece, si farà viva l'idea di identità di istriano durante i gravi rivolgimenti del terribile decennio che va dal ' 45 al ' 55 , quando la gente del suo paese fu forzata a dover scegliere un'identità. Durante il governo jugoslavo si voleva che tutti fossero slavi e comunisti, e soprattutto poveri e contrari ad ogni istituzione precedente, perfino alla Chiesa. ${ }^{7} \mathrm{E}$ in quel momento il mondo cominciò a dividersi per appoggiare il regime, e chiunque aveva il diritto di fare quello che voleva, persino di denunciare il vicino di casa solo perché italiano. Questi fatti vengono illustrati in maniera lucida in Materada, quando il protagonista, Francesco Coslovich, si reca dal vecchio zio barba Nin per

5 «Le popolazioni dell'interno amavano il proprio dialetto, con ingredienti volgarizzati veneti e croati, nella cui singolare parlata viveva, con valori trasmessi di generazione in generazione, l'antica onestà di un piccolo mondo rurale. Gli uni e gli altri si scambiavano gli attrezzi agricoli, si aiutavano, nel clima di solidarietà tipico della gente semplice dei campi, persone che amavano i fatti concreti e la parola data. Costoro comunicavano usando due dialetti, e si capivano superando qualsiasi barriera. Anche i giovani che si sposavano lo facevano senza distinzione di etnia, fosse essa italiana o croata» (ivi, p. 140).

6 Ivi, p. 124.

7 La chiesa, per i poveri contadini di Materada, era una delle istituzioni più importanti del paese, «il loro primo punto di riferimento», come dirà Tomizza. Ad essa erano legate tutte le loro festività, le ricorrenze (battesimi, matrimoni, morte), ma anche le loro preghiere, i loro valori tradizionali. F. Tomizza, Alle spalle di Trieste, cit., p. 140. 
chiedergli consiglio su cosa fare, se partire o meno per Trieste; i due parlano dei fatti accaduti in quei giorni e il vecchio, riferendosi a ciò che stava accadendo, dice: «Qui non c'è legge, non c’è ordine: il primo che si alza la mattina ha il comando nelle mani. Devi fare quello che vogliono loro; e come colono non ti resta altro da fare». ${ }^{8}$ Le parole di barba Nin sono molto dure verso coloro che detengono il potere, ch'egli definirà addirittura 'lupi', contro i quali solo i più forti sapranno difendersi, e specificherà che durante il periodo austriaco erano comunque tempi diversi, quando la giustizia era garantita dalla legge e alla domanda che gli pone Francesco se anche al tempo dell'Austria ci fossero queste discordie etniche, barba Nin risponde che anche allora ci si schierava da una e dall'altra parte per motivi etnici, ma secondo lui non lo si faceva per interesse, e comunque su tutto vigilava la legge:

«Chiedilo a tuo zio; chiedilo a quel disonesto. Io da una parte, lui dall'altra. Io italiano, lui croato. Io con la mia bandiera e i miei della Lega, lui con la sua e i suoi del Društvo. L'Austria permetteva. Ma quei bastardi ci accusarono di aver dato fuoco alla loro scuola. No, gli dico, signor giudice: questo no. Tutto si può dire (che li abbiamo presi a sassate, che mio fratello Zorzi ha fatto i suoi bisogni sulla bandiera, che quando tornavano da Babici erano in troppi e allora non mi restò altro da fare che ordinare la ritirata e, prima di darmela a gambe anch'io, calare le brache e mostrargli una cosa, signor giudice) ma non che noi abbiamo dato fuoco alla loro scuola! Non siamo mica venuti dalla Serbia, noi. Chiedete, informatevi: la scuola l'hanno bruciata le loro donne, che facevano il forno per cuocere il pane, non io, non i miei, signor giudice. E mi credette sulla parola».

«Dunque anche quella volta c'era la solita storia di italiani e slavi, slavi e italiani?»

Allora tuonò. «Ma quella volta si poteva! Il mondo andava così, e in fondo era anche un divertimento: mai un gioco di interessi. Quella volta si ballava anche la mazurca, ma va a vedere al Dom a quanti dei nostri giovani piace ballarla!» ${ }^{9}$

Dalle parole del vecchio si intravede che egli apprezzava il governo austriaco in quanto rispettava le diversità consentendo la convivenza, ma nel periodo in cui si svolgono i fatti narrati in Materada (quello dell'avvento del governo jugoslavo) la situazione era molta diversa, densa di un odio che porta alla dolorosa vicenda dell'esodo. Anche in questo ambito si può intravedere il problema della nazionalità nelle popolazioni di frontiera, che spesso è vissuta come un dramma, specialmente quando si tratta di dover odiare o (peggio) combattere chi si è sempre visto come amico, anche se di altra nazionalità.

Un importante dramma che nasce nelle popolazioni istriane, legate alla realtà contadina quasi come unico simbolo di riconoscimento, consiste nel fatto

8 Fulvio Tomizza, Materada, Milano, Bompiani, 2000, p. 123.

9 Ivi, p. 127. 
di dover lasciare la propria terra, considerata come il luogo sacro del lavoro. Il protagonista di Materada, infatti, non voleva abbandonarla perché vi era legato da un vincolo molto forte, affettivo, e non riusciva nemmeno a immaginare come sarebbe stata la vita in città, lontano dai suoi campi, dal suo lavoro. E il giorno in cui decise di farlo, di optare per l'Italia e pertanto lasciare la propria terra, fu un momento difficile, tragico. All'inizio non gli sembrava una cosa molto grave, avrebbe firmato dei documenti e tutto sarebbe finito lì, ma in verità, quando si recò a Umago per andare a firmare i documenti, mentre saliva le scale che lo avrebbero portato all'ufficio per la firma, si sentì mancare, poi si ricordò che a Trieste nel campo profughi gli avrebbero dato «una baracca, due pasti, il sussidio» e allora i suoi pensieri ritornarono alla sua terra, al lavoro che non avrebbe più avuto, alle festività e alle ricorrenze della sua gente e a cosa avrebbe potuto comprarsi con i guadagni del sudore del suo lavoro. ${ }^{10} \mathrm{Nel}$ momento della decisione, quando Francesco si reca a Umago per firmare la richiesta di partire, i ricordi vanno alla terra, a quel mondo che deve lasciare e al fatto che non riusciva a immaginarsi una nuova vita in cui non ci fosse questa sua realtà. Il contadino di Materada nell'atto di varcare il confine che lo esclude per sempre dai suoi campi e dalle sue stalle, si volta indietro e prova «un certo scherzo: era come se l'amico, dentro, avesse per un momento cessato di battere. Davanti a me vedevo un mare aperto, nel quale si doveva entrare; e non sapevo come, né da che parte incominciare». ${ }^{11}$

La terra esprime la vera natura dell'identità di frontiera perché rimane il punto di riferimento, una certezza, in un certo senso eterna e irremovibile, per l'individuo; attraverso di essa, anche se plasmata dai vari eventi storici, si mantiene la stabilità, permettendo all'individuo di relazionarsi ad essa in quanto immutabile nella sua essenza. Per il protagonista tomizziano l'unica appartenenza certa è quella alla terra natale, che permette di identificarsi e riconoscersi in essa. $\hat{\mathrm{E}}$ per questo motivo che probabilmente Tomizza fa concludere Materada con la riconciliazione in chiesa prima di partire. È un atto di coraggio, quello che s'impongono i paesani, di dire la messa da soli senza il parroco (che, per i tempi che corrono, in paese non hanno più) per poter lasciare la loro terra in pace. E così il romanzo si conclude con le parole del protagonista, Francesco, che pensa alla propria terra, fonte di vita, e alla moltitudine dei morti nati e cresciuti e rimasti lì, una terra che avrebbe potuto bastare per loro e per i loro figli. ${ }^{12}$

10 Ivi, p. 142.

11 Ivi, p. 145.

12 Ivi, p. 173. 
Questi sono i valori di un mondo contadino: la terra, la chiesa, i morti, il cimitero con il confine che va forzatamente varcato e porterà così a una lacerazione profonda tra ambiente umano e naturale dovuta al distacco dell'esilio. ${ }^{13}$

\section{Identità di frontiera}

La definizione di identità è strettamente legata alle componenti presenti e passate di una specifica cultura, caratteristiche che sono l'effetto di un processo precario, in cui le circostanze storiche cambiano nel tempo, aggiungendo o togliendo specificità culturali ed etniche. L'Istria è una regione di natura multietnica con un forte spirito di convivenza con la diversità in cui le definizioni identitarie imposte dai vari regimi hanno creato invece ostilità. Le varie vicissitudini storiche del territorio hanno portato a spostare nel tempo le diverse linee di confine, e questo, di fatto, ha contribuito alla creazione di un territorio multietnico e multilingue:

Chi nasce in quel territorio è ricco di tante differenze e di tante culture, è se stesso con un'identità netta o predominante, ma è anche l'altro, ha in sé anche un po' dell'altra identità. Ogni tanto, la Storia impone alla gente di frontiera di avere una sola identità. E questo è sempre doloroso, talvolta tragico. Talvolta si arriva alla soluzione barbara e parossistica del trasferimento di popolazioni (inutile fare scempi) nella ricerca demente di una sola identità. ${ }^{14}$

Il vincolo causato da una scelta politica significa l'esigenza di nominare, assieme all'ideologia, una delle due identità etniche e culturali presenti negli individui, ponendo la necessità di scegliere tra una e l'altra, dilemma questo al quale è

13 Con Materada lo scrittore ha voluto fare quasi un tributo alla sua terra, alla sua gente che è stata sballottata dalla storia, dagli eventi bellici, dalle epidemie. Sentiva di dover lasciare una testimonianza e l'ha fatto con i suoi romanzi, iniziando proprio da questo che molti critici considerano ancora il suo romanzo più bello. Molte delle sue opere sono ambientate nel periodo tra il 1945 e il 1955 che è stato il periodo storico più sconvolgente dell'Istria. Da ragazzo lo scrittore ne è stato testimone e l'ha vissuto in prima persona, per cui nei primi libri ha cercato di testimoniare e specialmente di portare una parola di pace, una parola di solidarietà, una parola nuova in un mondo che era secolarmente sempre pieno di conflitti, di rancori e di lacerazioni. 14 La citazione riporta le parole del regista triestino Franco Giraldi che parla della sua concezione della frontiera. Il passo è tratto dall'articolo di Laura Strano, Giraldi: la frontiera, il mio tormento di bambino, uscito su «Il Piccolo» del 31 luglio 2006 in cui viene presentato il film del regista La frontiera tratto dal romanzo omonimo di Franco Vegliani. 
impossibile dare una soluzione, o per lo meno una soluzione che non porti sofferenza. La prospettiva che propone di adottare Tomizza è quella di vedere il rapporto con il diverso come un arricchimento ed un'apertura; un tema, questo, che si trova in molti dei suoi romanzi.

Riprendendo le parole del sociologo Ulderigo Bernardi potremmo affermare che Tomizza sia riuscito a descrivere quelli che sono i tratti essenziali dell'identità dell'Istria (o se vogliamo di un'identità di frontiera), per cui questa terra è

intesa come un abecedario spalancato sulle culture. Uno scenario estroso, scabro e armonico, dove le asprezze carsiche sfumano nella dolcezza delle doline, e l'eleganza di umili architetture rurali rispecchia l'urbanità dei centri costieri [...] Chi visita l'Istria compie un pellegrinaggio di memoria, per i molti segni di patrie perdute che questa terra conserva. Ma al tempo stesso avverte la percezione di camminare lungo la fresca via del mattino d'una umanità che avrà in orrore le prigioni, etniche o d'altro genere, mentre vive sommessamente ogni giorno, nella quotidianità etica del lavoro e nella solidarietà comunitaria, la speranza tenace di aria nuova per le sue culture. Fulvio Tomizza muovendo dall'appartenenza locale guidava a riflettere sull'universalità perenne della mescolanza: la terra d'origine come tutta la Terra, e la cultura universale come universo di culture. Ciascuna degna di rispetto. ${ }^{15}$

La concezione della frontiera delineata da Tomizza si trova, oltre che nei romanzi, anche in altre sue opere, quali conferenze, articoli e discorsi apparsi in varie circostanze e raccolti in Alle spalle di Trieste (con scritti che vanno dal 1969 al $1994)^{16}$ e in Destino di frontiera (1992), una lunga intervista di Riccardo Ferrante, scrittore triestino di origine istriana, a Tomizza. In questi volumi vengono ripresi e spiegati direttamente dall'autore i temi principali di molti suoi romanzi, tra i quali emerge quello dell'identità di frontiera.

Tomizza è stato considerato da diversi critici lo scrittore di frontiera per eccellenza; ${ }^{17}$ egli ha vissuto un doppio esilio, istriano e triestino, ed è stato sempre particolarmente sensibile alle sofferenze e ai problemi del vivere quotidiano, di chi, come lui, è uomo di due patrie. È proprio negli interventi che possiamo leggere nei due libri analizzati che lo scrittore si rivela anche storico della propria esperienza e fa luce su una fase della sua vita: quel periodo travagliato della storia che è rimasto impresso nella sua memoria. ${ }^{18}$ Lo scrittore

15 Ulderico Bernardi, Istria d'amore. L'Istria, magico frammento d'Europa, Treviso, Santi Quaranta, 2012, p. 137.

16 In molti degli articoli del libro lo scrittore affronta i problemi della frontiera.

17 Antonino Grillo, Tomizza e la critica più recente: a proposito di Carmelo Aliberti. Fulvio Tomizza e la frontiera dell'anima, Foggia, Bastogi, 2003.

18 In un'intervista lo stesso scrittore affermerà: «Si vede che la mia penna non scrive bene, non si muove se non consola qualche ferita ingiusta, se non asciuga qualche lacrima, se non porta 
tratta questi temi per non far dimenticare quel periodo atroce, ma soprattutto perché vorrebbe che queste terre (e qui pensa anche a Trieste) vivessero quella che lui considerava la riconciliazione tra etnie. Lo scrittore avrebbe voluto fermare il «contrasto irriducibile» che c'era tra la gente di questa regione, per attuare «l'impossibile riconciliazione», in modo che non si dovesse più fare una scelta tra culture e etnie diverse, «ma tentando piuttosto di accordarle, riconoscendole proprie di un uomo di frontiera, sentendole stimolanti anziché gravose». ${ }^{19}$

\section{Tra utopia e disillusione}

Tomizza arrivò a Trieste dall'Istria nel 1955, trovando una città in un certo senso ostile, avversa, anche perché in essa si concentravano i conflitti e le lacerazioni che lui in parte aveva già superato. ${ }^{20} \mathrm{Da}$ una parte si sentiva attratto verso il mondo cittadino urbano, borghese, verso la cultura supponente che aveva dei riferimenti tanto precisi in Saba, Svevo, Slataper; dall'altra parte questa stessa città gli provocava come un senso di soffocamento ed è per questo che sente il bisogno di uscire e di respirare l'aria dell'Istria, ed è in questo frangente che, come alternativa all'Istria, si pone un nuovo paesaggio campestre, molto singolare: il Carso triestino.

Tomizza descrive il suo arrivo e la sua vita a Trieste nel romanzo L'amicizia, in cui racconta la storia di due uomini e di due situazioni ambientali: Trieste e il Carso. I protagonisti del romanzo sono un giovane di campagna arrivato in città, Marco (l'alter ego dello scrittore), e Alessandro, un ragazzo di città. Tra i due si sviluppa un rapporto di amicizia che si trasforma via via in un confronto continuo di caratteri e spesso sfocia in un provocatorio scambio di ruoli.

sollievo, calore, compagnia a persone che non ne hanno avuta. Io ho sofferto di solitudine e so quanto fa bene una parola di consolazione e affetto» (Robert Apollonio, Tomizza e i suoi romanzi. TRV Koper-Capodistria, 28.4.2009).

19 F. Tomizza, Alle spalle di Trieste, cit., p. 143.

20 Fulvio Tomizza era arrivato a Trieste in seguito all'esodo e negli anni ' 60 aveva già pubblicato i suoi primi libri su questo argomento. Il richiamo alla lacerazione superata si riferisce al fatto che lo scrittore aveva capito già precedentemente che per poter vivere in armonia in terre di confine, bisognava accettare la presenza di tutte le etnie e le culture presenti nel territorio, cosa che invece non trova a Trieste, perché è molto forte ancora il sentimento di disprezzo nei confronti degli sloveni presenti sul territorio. 
Marco, come lo scrittore, per sfuggire alla sua sofferenza in città andava proprio sul Carso, al fine di trovarsi in uno spazio più aperto, più respirabile, più congeniale. Nel Carso egli trova qualcosa di veramente interessante, ossia delle similitudini con l'Istria, ma rispetto al suo paese istriano, questo ambiente era più aspro, più chiuso, con una popolazione totalmente slovena che però gli ricordava il mondo arcaico in cui aveva trascorso l'infanzia. E pertanto era naturale che «quel luogo, quelle stradine, quei muri, quei roveri» ${ }^{21}$ gli parlassero più che a un triestino che da Trieste arrivava sul Carso per una passeggiata:

Ai tratti di bosco succedevano sempre più frequenti ed estesi i campetti di terra rossa tenuti a viti alte, sorrette da grossi pali di acacia, mentre alla mia destra il sottobosco biancastro per le rocce affioranti si spingeva a vestire i poggi in corsa ondulata con noi.

Il cuore mi diede un sobbalzo. Mi sbirciasti per azzardare: «Qui mi pare di ritrovare il tuo paesaggio».

«Infatti» risposi in piena emozione. «È il punto che maggiormente assomiglia ai miei luoghi. Mi basterebbe chiudere gli occhi e sentire le erte improvvise della strada».22

A contatto con la gente del Carso, si sentiva come una specie di fratello, parlava con loro in sloveno e quindi veniva considerato uno della famiglia. Al contrario Alessandro, il personaggio che lo accompagna, è il suo opposto, è l'amico cittadino che va sul Carso con una specie di pregiudizio, di distacco, nonostante sia un personaggio molto aperto, decisamente cosciente delle sue scelte. Egli guarda alla gente del Carso con un certo distacco e, proprio per questo motivo, il suo amore con Irena, la ragazza slovena, non si realizza.

Rispetto ai libri precedenti, in questo romanzo Tomizza esprime tutta la sua amarezza nei confronti di fatti non avvenuti. ${ }^{23}$ Prima l'amicizia che non si realizza completamente tra il ragazzo di campagna istriano, con i suoi orizzonti aperti, e il ragazzo di città, aperto al colloquio e al dialogo, però segretamente condizionato dalla chiusura triestina, cittadina. Così come non si realizza neppure il rapporto amoroso tra Alessandro e Irena, la fidanzata slovena.

Con questo romanzo Tomizza ha voluto esprimere la disillusione rispetto alla sua utopia, all'illusione di aver creduto che ci fosse un processo di osmosi tra i popoli al di qua e al di là del confine, il conseguente superamento di ogni sospetto, di ogni intolleranza. Al contrario, con L'amicizia, Tomizza ha voluto

21 Fulvio Tomizza, L'amicizia, Milano, Rizzoli, 1980, p. 143.

22 Ivi, p. 139.

23 Lo scrittore era dilaniato da un conflitto interiore, perché nel clima della guerra fredda si rendeva conto che era impossibile la realizzazione del suo sogno di superare i contrasti tra la cultura italiana e la cultura slava, che egli sentiva entrambe presenti nella sua identità di uomo di frontiera. 
esprimere il suo rammarico per essere stato «un cattivo profeta», ${ }^{24}$ lui stesso vivrà una sconfitta morale e personale, realizzando che la barriera che aveva creduto superata, non solo persisteva ma era ancora molto forte, molto sentita, sia da una parte che dall'altra.

Nel momento in cui Tomizza scrive il romanzo forse non c'è inimicizia, non c'è odio, ma c'è freddezza: in un mondo di confine come questo, nel quale ognuno ha bisogno dell'altro, non ci dovrebbe essere freddezza. Lo scrittore ci vuol dire che in un territorio come il Friuli Venezia Giulia e l'Istria ci deve essere quell'apertura e quella disposizione dell'anima libera da pregiudizi per cui si può lavorare insieme per fare qualcosa di nuovo. Secondo Tomizza è mancata la volontà di collaborazione, e questo viene reso palese nel titolo L'amicizia, un rapporto bello, umano, forse il più umano dei rapporti che si può costituire libero senza alcuna scelta tra due persone. Altrettanto bello sarebbe il rapporto schietto, di incontro tra due popolazioni, che in certi momenti sembra avvenire, per poi invece cessare improvvisamente per il prevalere del pregiudizio in entrambe le etnie, quella italiana e quella slava. ${ }^{25}$

\section{Conclusioni}

Tomizza, attraverso le sue opere, cerca di trasmettere un processo di armonizzazione tra le due componenti etniche e culturali presenti in Istria. Per presentare al meglio i suoi concetti, lo scrittore usa uno stile introspettivo e autobiografico, che rafforza l'argomentazione della sua tesi di convivenza e armonia delle diverse culture. Argomento questo che ci riporta a Materada in cui lo scrittore ricorda l'armoniosa vita che viveva la comunità prima dei terribili fatti storici. Le popolazioni dell'interno amavano il proprio dialetto, con ingredienti volgarizzati veneti e croati, nella cui singolare parlata viveva, con valori trasmessi di generazione in generazione, l'antica onestà di un piccolo mondo rurale. Gli uni e gli altri si scambiavano gli attrezzi agricoli, si aiutavano, nel clima di solidarietà tipico della gente semplice dei campi, persone che amavano i fatti concreti e la parola data. Costoro comunicavano usando due dialetti, e si capivano superando qualsiasi barriera. Anche i giovani che si sposavano lo facevano senza distinzione di etnia, fosse essa italiana o croata; ricordiamo che la stessa madre dello scrittore Fulvio Tomizza era di origine slovena, ed era

24 Robert Apollonio, Tomizza e i suoi romanzi, cit.

25 Ibid. 
sposata con un italiano. ${ }^{26}$ Tomizza riconosce la propria appartenenza ad entrambi i mondi, quello italiano e quello slavo, ${ }^{27}$ e la racconta nelle sue opere, presentando un resoconto realistico di esperienze vissute.

Secondo Tomizza l'unica soluzione per giungere alla riconciliazione di queste due realtà è il risorgimento di quella specifica caratteristica che lui ascrive al suo territorio natale: il riconoscimento e l'assimilazione delle diversità. Per questo motivo lui stesso è posto davanti a una scelta che limiterebbe il suo essere: non poteva accettare una sola realtà culturale e etnica, ma l'unica cosa che poteva fare era quella di considerarle insieme come un'unica identità. ${ }^{28}$

Anche il sociologo Darko Bratina, amico e collaboratore di Tomizza, con cui lo scrittore ha condiviso l'amore per il cinema, facendo riferimento alla questione dell'identità di frontiera si esprime in questi termini:

Personalmente pure essendo sloveno di lingua e di cultura, mi sentirei privato di molto se non potessi muovermi normalmente nella lingua e nella cultura italiana e tra italiani, non solo perché mi ritengo cittadino italiano a tutti gli effetti, ma perché senza questa non presenza capirei ben poco del luogo in cui vivo e della gente con cui convivo e incontro quotidianamente.

Per quel che mi riguarda essa mi permette di vivere nella sua pienezza, e non da straniero il luogo fisico e antropologico che la storia in un certo senso ha imposto quasi come un dato di natura. Non solo ma, oltre a trovarmi a casa qui sulla frontiera, mi trovo perfettamente a casa sia a Milano che a Lubiana, nel senso che la gente ed il contesto complessivamente non mi sono né lontani né stranieri ed interagisco con entrambi. Pertanto essere insieme sloveno e italiano non è difficile semmai è solo affascinante. ${ }^{29}$

Secondo Tomizza l'Istria rimane una regione multietnica nella quale si incrociano e convivono, spontaneamente, un vasto numero di culture. La popolazione originaria della regione è custode di un'identità plurima, un miscuglio etnico e culturale che si è sedimentato con l'andare del tempo e che ha generato una dimensione umana tollerante e aperta.

26 F. Tomizza, Alle spalle di Trieste, cit., p. 190.

27 «La duplicità che mi porto addosso non credo si chiamasse doppiezza. Era un instabile e sofferto coesistere di due modi di essere e di sentire contrapposti, due appartenenze che non riuscivano a conciliarsi e s'incolpavano a vicenda...» (Fulvio Tomizza, Il sogno dalmata, Milano, Mondadori, 2002, p. 44).

28 «Nessuna delle due fazioni mi aveva interamente. Esse esistevano nella mia immaginazione soltanto se a vicenda si completassero o si scambiassero qualcosa: la lingua innanzitutto, e poi l'istruzione e l'urbanità da un lato, l'insicurezza, l'umiltà, la voglia di fare e di cambiare il mondo dall'altro» (ivi, p. 45).

29 Palčič, Boris, Et(h)nos. Anche noi. Tudu mi, film documentario, Kinoatelje, Gorizia, 2004. 
Lo scrittore si augura, come descrive nelle sue opere, che l'Istria diventi «da un luogo di continui attriti, la frontiera che può rovesciarsi in oasi di pace». ${ }^{30} \mathrm{E}$ questo il messaggio dell'autore che, anche se può sembrare troppo ottimista e quasi utopico, propone l'unica soluzione auspicabile per il futuro non solo dell'Istria, ma anche dell'Europa stessa. ${ }^{31}$

Negli ultimi anni della sua vita, quando capì che la sua utopia sembrava condivisa dalla maggioranza dei suoi conterranei, stanchi di odiarsi l'un l'altro, lo scrittore ha addirittura azzardato l'idea di una regione autonoma istriana che rispettasse la sovranità territoriale dei tre Stati governanti (Italia, Slovenia e

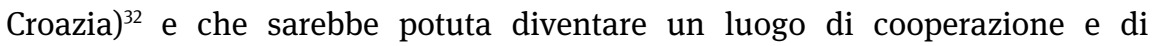
convivenza, di approccio inventivo, di scambio di esperienze, di mutuo ricorso ad altre culture, di scoperte e rivisitazioni.

30 F. Tomizza, Alle spalle di Trieste, cit., p. 194.

31 Fulvio Tomizza, Le mie estati letterarie. Lungo le tracce della memoria, Venezia, Marsilio, 2009, p. 57-59.

32 Ivi, p. 214-215. 
